\title{
DIVISION BY HOLOMORPHIC FUNCTIONS AND CONVOLUTION EQUATIONS IN INFINITE DIMENSION
}

BY

\author{
J. F. COLOMBEAU ${ }^{1}$, R. GAY AND B. PERROT
}

\begin{abstract}
Let $E$ be a complex complete dual nuclear locally convex space (i.e. its strong dual is nuclear), $\Omega$ a connected open set in $E$ and $\delta(\Omega)$ the space of the $C^{\infty}$ functions on $\Omega$ (in the real sense). Then we show that any element of $\mathcal{E}^{\prime}(\Omega)$ may be divided by any nonzero holomorphic function on $\Omega$ with the quotient as an element of $\mathcal{E}^{\prime}(\Omega)$. This result has for standard consequence a new proof of the surjectivity of any nonzero convolution operator on the space $\operatorname{Exp}\left(E^{\prime}\right)$ of entire functions of exponential type on the dual $E^{\prime}$ of $E$. As an application of the above division result and of a result of $C^{\infty}$ solvability of the $\bar{\partial}$ equation in strong duals of nuclear Frechet spaces we study the solutions of the homogeneous convolution equations in $\operatorname{Exp}\left(E^{\prime}\right)$ in terms of the zero set of their characteristic functions.
\end{abstract}

1. Statement of the results. In this section $E$ denotes a complex quasi-complete dual nuclear locally convex space (i.e. its strong dual is a nuclear l.c.s.) and $\Omega$ an open subset of $E$. Considering naturally $E$ as a real vector space we define the $C^{\infty}$ functions $f$ on $\Omega$ in the following way: $f: \Omega \rightarrow \mathbf{C}$ is $C^{\infty}$ if for every convex balanced bounded subset $B$ of $E$ the restriction of $f$ to $\Omega \cap E_{B}$ (where $E_{B}$ denotes as usual the vector space spanned by $B$ and normed with the gauge of $B$ ) is $C^{\infty}$ in the usual Fréchet sense of calculus in normed spaces. In the above case for $E$ this definition is that of the Silva $C^{\infty}$ functions on $\Omega$. Since $E$ is dual nuclear we are going to see that each bounded subset $K$ of $E$ is in fact relatively compact in some normed space $E_{B}$ where $B$ is a suitably chosen convex balanced bounded subset of $E$; we say in this case that $K$ is "strictly relatively compact"; hence each compact subset of $\Omega$ is "strictly compact" (i.e. compact in some $\Omega \cap E_{B}$ ). We equip $\mathcal{E}(\Omega)$ with the topology of uniform convergence of the functions and all their derivatives on each compact ( = strictly compact) subset of $\Omega$ : a base of 0 -neighborhoods of $\mathcal{E}(\Omega)$ is made of the sets

$$
\mathcal{V}(K, L, \varepsilon, n)=\left\{f \in \mathcal{E}(\Omega) \text { such that } \sup _{\substack{x \in K \\ y_{j} \in L}}\left|f^{(i)}(x) y_{1} \ldots y_{i}\right| \leqslant \varepsilon \text { if } 0 \leqslant i \leqslant n\right\}
$$

where $K$ is a variable compact subset of $\Omega, L$ a variable bounded (or equivalently compact) subset of $E, \varepsilon>0$ and $n \in \mathbf{N}$.

Received by the editors December 11, 1979.

AMS (MOS) subject classifications (1970). Primary 46G20, 35R15; Secondary 32A10, $35 E 99$.

Key words and phrases. Infinite dimensional holomorphy, infinite dimensional distribution, convolution equation, $\bar{\partial}$ equation, Fourier-Borel transform.

${ }^{1}$ The research of this author was supported in part by the "Financiadora de Estudos e Projetos" and the "Fundacão de Amparo a Pesquisa do Estado de São-Paulo" during his stay at the Universidade Estadual de Campinas (Brazil) in the local winter term 1978. 
REMARK 1. There are a lot of various definitions of $C^{\infty}$ functions on locally convex spaces (see Averbuck and Smolyanov [1], Nashed [18], Colombeau and Meise [6]) but, in this above case for $E$, the spaces of the "natural" concepts of $C^{\infty}$ functions on $\Omega$ are dense in $\mathcal{E}(\Omega)$ (see Colombeau and Meise [6]). Since we only use $\mathcal{E}(\Omega)$ by its dual $\mathcal{E}^{\prime}(\Omega)$ we see that we do not use in fact our particular concept of $C^{\infty}$ functions (that we have chosen because it is convenient) and that our results are valid for "most" of the concepts of $C^{\infty}$ functions in locally convex spaces.

Recalling now that $E$ is a complex vector space we define a Silva-holomorphic function $f$ on $\Omega$ by: for every convex balanced bounded subset $B$ of $E$ the restriction of $f$ to $\Omega \cap E_{B}$ is holomorphic in the sense of normed spaces. In particular every $G$-analytic and continuous function is Silva-holomorphic.

We obtain

THEOREM 1. Let $E$ be a complex quasi-complete dual nuclear l.c.s. and $\Omega$ a connected open subset of $E$. Let $\rho$ be a nonzero Silva-holomorphic function on $\Omega$. Then for every element $T$ of $\mathcal{E}^{\prime}(\Omega)$ there exists an element $S$ of $\mathcal{E}^{\prime}(\Omega)$ such that $\rho S=T$.

Theorem 1 generalizes a finite dimensional result of Schwartz [21] and the proof given in the sequel is also a generalization of Schwartz's proof.

REMARK 2. It is not known whether one may replace $\rho$ in Theorem 1 by a nonzero real polynomial on $E$ and more generally by a nonzero real analytic function on $\Omega$ (in the finite dimensional case these are famous results of Hörmander [16] and Lojasiewicz [17] respectively).

Let us denote now by $\mathscr{F}$ the Fourier-Borel transform and let us assume that $\Omega$ is convex balanced. Let us equip the space $\mathcal{H}_{S}(\Omega)$ of the Silva-holomorphic functions on $\Omega$ with the topology of the uniform convergence on the strictly compact subsets of $\Omega$ and the space $\mathcal{H}(\Omega)$ of the holomorphic (i.e. $G$-analytic and continuous) functions on $\Omega$ with the compact open topology. Then since $E$ is a quasi-complete dual nuclear l.c.s. (Colombeau, Meise and Perrot [7]) $\mathcal{H}(\Omega)$ is dense in $\mathcal{H}_{S}(\Omega)$ and they have the same dual denoted by $\mathcal{H}^{\prime}(\Omega)$. The Fourier-Borel transform is injective and we equip $\mathscr{F} \mathcal{H}^{\prime}(\Omega)\left(\subset \mathcal{H}\left(E^{\prime}\right)\right)$ with the image via $\mathscr{F}$ of the strong topology of $\mathcal{H}^{\prime}(\Omega)$.

Remark 3. The space $\mathscr{F} \mathcal{H}^{\prime}(E)$ is described, as usual, in Boland [2], [3] as a space of entire functions of exponential type on $E^{\prime}$, more precisely:

$\mathscr{F} \mathcal{H}^{\prime}(E)$ is the vector space of the holomorphic functions $\phi$ on $E^{\prime}$ such that there is a compact subset $B$ of $E$ and $c>0$ with:

$$
|\phi(\xi)| \leqslant c e^{|\xi|_{B}} \quad \forall \xi \in E^{\prime}
$$

with $|\xi|_{B}=\sup _{X \in B}|\xi(x)|$. Denote by $\mathscr{B}(B, c)$ the set of the functions $\phi$ satisfying the above inequality and by $\mathcal{E}(B, c)$ the vector span of $\mathscr{B}(B, c)$ equipped with the Minkowski functional of $\mathscr{B}(B, c)$. Then $\mathscr{F} \mathcal{F C}^{\prime}(E)$ is equipped with the locally convex inductive limit of the normed spaces $\mathcal{E}(B, c)$. In this way $\mathscr{F} \mathcal{F}^{\prime}(E)$ is topologically isomorphic (via the Fourier-Borel transform $\mathscr{F}$ ) with $\mathcal{H}^{\prime}(E)$ equipped with the strong dual topology. We denote as usual $\mathscr{F} \mathcal{H}^{\prime}(E)$ by $\operatorname{Exp}\left(E^{\prime}\right)$.

As usual we define a convolution operator on $\operatorname{Exp}\left(E^{\prime}\right)$ as a linear continuous map from $\operatorname{Exp}\left(E^{\prime}\right)$ into itself which commutes with the translation $\tau_{\alpha}$ for any $\alpha \in E^{\prime}$. 
TheOREM 2 (EXISTENCE OF SOLUTIONS). Let E be a complex quasi-complete dual nuclear l.c.s. Then any nonzero convolution operator on $\operatorname{Exp}\left(E^{\prime}\right)$ is surjective.

The proof given in the sequel is a standard consequence of Theorem 1. It is completely different from the proof given in Colombeau and Perrot [9] where Theorem 2 was already obtained.

Now, as a consequence of Theorem 1 and of the result of existence of $C^{\infty}$ solutions for the $\bar{\partial}$ equation in DFN spaces proved in Colombeau and Perrot [10] one obtains the following result which generalizes part of the results in Gay [13].

TheORem 3. Let $E$ be a complex quasi-complete dual nuclear l.c.s. Let $\mathcal{O}$ be a convolution operator on $\operatorname{Exp}\left(E^{\prime}\right)$ and let $\rho$ in $\mathcal{H}_{S}(E)$ be its characteristic function. Then any solution $f$ in $\operatorname{Exp}\left(E^{\prime}\right)$ of the homogeneous equation $\theta f=0$ is the FourierBorel transform of an element $U$ of $\mathcal{E}^{\prime}(E)$ such that $\rho U=0$ (which implies, in most of usual cases for $E$, that supp $U$ is contained in the set of zeroes of $\rho$ ).

If $E$ is a DFN space the proof is a direct generalization of Gay's finite dimensional proof [13]. The general case for $E$ is more complicated but will be reduced to this particular case, using the technique developed in Colombeau and Perrot [9].

As previous results on a similar topic, let us quote the very serious works of Dwyer [11], [12] on differential equations of infinite order in locally convex spaces.

In fact Theorems 1, 2 and 3 may be stated in a more general setting using the so-called "convex bornological vector spaces" (b.v.s. defined in the pioneer article of Waelbroeck [22]). The more important fact is that the proofs of the theorems, even in the form they are stated above, use naturally this structure; hence it seems to us better for the transparency of them to define and use explicitly this structure.

In the next section we define and recall some known facts on these b.v.s. In the further sections we state the theorems in their general formulation and prove them.

2. A few concepts and results involving "bornology". As defined by Waelbroeck in [22], [23] (under the name of $b$-space) a convex bornological vector space $E$ (b.v.s. for short) is an algebraic injective inductive limit of normed spaces $\left(E_{i}\right)_{i \in I}$ ( $I$ is a directed set of indices) and by definition we say that a subset of $E$ is "bounded" iff it is contained in some normed space $E_{i}$ and bounded there. Two such structures on $E, E=\lim _{\rightarrow \in I} E_{i}$ and $E=\lim _{j \in J} F_{j}$ (algebraically) are identified if they have the same bounded sets. We denote by $E^{\times}$the bornological dual of $E$ (i.e. the vector space of the linear forms on $E$ that are bounded on each bounded subset of $E$; for short the "bounded linear forms"). We say that $E$ is $t$-separated if $E^{\times}$ separates $E$ : it is an easy consequence of the Hahn-Banach theorem that $E$ is $t$-separated iff the locally convex inductive limit $\lim _{i \in I} E_{i}$ is Hausdorff. This space $E^{\times}$is always equipped with the topology of uniform convergence on the bounded subsets of $E$.

A b.v.s. $E$ is said to be Schwartz (respectively nuclear) if there exists a representation $E=\lim _{i \in I} E_{i}$ where these spaces $E_{i}$ are Banach spaces and where for every

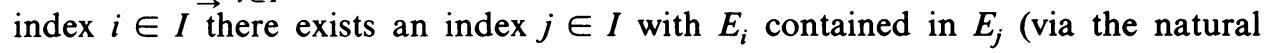
injection $\pi_{i j}: E_{i} \rightarrow E_{j}$ coming from the injective inductive system) and such that $\pi_{i j}$ 
is a compact (resp. a nuclear) map. If $E$ is a quasi-complete dual nuclear l.c.s. then it is known and easy to check that the von Neumann bornology of $E$ is a nuclear bornology, hence a fortiori a Schwartz bornology. Note that if $E$ is a Schwartz b.v.s. then any bounded subset is also strictly relatively compact (this fact is alluded to in $\$ 1$ ).

If $\Omega$ is a subset of the b.v.s. $E$ we say that $\Omega$ is $M$-open if for every index $i \in I$, $\Omega \cap E_{i}$ is open in the normed space $E_{i}$. The definitions of the (Silva) $C^{\infty}$ functions or (Silva) holomorphic functions on $\Omega$ are exactly those given in $\S 1$ (for the particular case of the von Neumann bornology of a l.c.s. $E$ ).

If $E$ is a b.v.s., a family $\left(c_{i}\right)_{i \in I}$ of bounded sets is called a "basis of bounded sets" if each bounded subset of $E$ is contained in some $c_{i}$.

\section{Division of infinite dimensional distributions by holomorphic functions.}

THEOREM 1 (GENERAL FORM). Let E be a complex $t$-separated Schwartz b.v.s. and $\Omega$ a connected $M$-open subset of $E$. Let there be given an element $T$ of $\mathcal{E}^{\prime}(\Omega)$ and a nonzero Silva-holomorphic function $\rho$ in $\Omega$. Then there exists an $S$ in $\mathcal{E}^{\prime}(\Omega)$ such that $\rho S=T$.

The end of this section is devoted to the proof of Theorem 1. Owing to a result of Ramis [20] the proof is a generalization of the finite dimensional proof of Schwartz [21].

We first recall a lemma which is Corollary (c), p. 14 of Theorem 1.1.3.4 of Ramis [20].

Lemma 1. Let $E$ be a complex Banach space, $\Omega_{1}$ an open subset of $E$ such that $0 \in \Omega_{1}$ and $g$ a nonzero element of $\mathcal{H}\left(\Omega_{1}\right)$ with $g(0)=0$. Then there exist a decomposition of $E$ in a topological direct sum $E=F \oplus \mathbf{C e}(e \neq 0)$, an invertible holomorphic function $J$ in a neighborhood $\mathcal{U}$ of the origin of $E$ and a polynomial

$$
P(z, \xi)=z^{p}+a_{1}(\xi) z^{p-1}+\cdots+a_{p}(\xi)
$$

where $z \in \mathbf{C}, \xi \in F$, the functions $a_{i}$ are holomorphic in $\mathscr{U} \cap F$ and $a_{i}(0)=0$ and such that, if $x=\xi+z e$ is in $\mathscr{U}$ :

$$
g(x)=P(z, \xi) J(x) .
$$

Now we need an infinite dimensional formulation of Lemma 3 of Schwartz [21, p. 186].

Let $\Lambda$ be a 0 -neighborhood in a real normed space $F$ (of norm \|\|$_{F}$ ); let $t$ denote a variable point ranging over $\Lambda$; let $\omega$ be a 0 -neighborhood in $\mathbf{C} \simeq \mathbf{R}^{2}$ and let $z$ denote a variable point ranging over $\omega$. Let

$$
P(z, t)=z^{p}+a_{1}(t) z^{p-1}+\cdots+a_{p-1}(t) z+a_{p}(t)
$$

where the functions $a_{i}$ are in $\delta(\Lambda)$ and such that all their derivatives are bounded in $\Lambda$.

Let

$$
\alpha_{m}=\sup _{\substack{1<i<p \\ 0<j<m \\ t \in \Lambda,\left\|t_{k}\right\|_{F}<1}}\left|a_{i}^{(j)}(t) t_{1} \ldots t_{j}\right| .
$$


Let $\varphi$ be in $\delta(\omega \times \Lambda)$ and let $\Psi=P \varphi$. Let $\omega^{\prime}$ be an open bounded 0-neighborhood in $\mathbf{C}$ such that its closure $\bar{\omega}^{\prime}$ is contained in $\omega$.

If $\theta$ is in $\mathscr{E}(\omega \times \Lambda), h$ and $k \in \mathbf{N}, r=\left(r_{1}, r_{2}\right) \in \mathbf{N}^{2}$ let

$$
M_{h, k}\left(\theta, \omega^{\prime} \times \Lambda\right)
$$

\section{LEMMA 2. With these notations}

$$
M_{h, k}\left(\varphi, \omega^{\prime} \times \Lambda\right) \leqslant B\left(h, k, p, \alpha_{k}, \omega, \omega^{\prime}\right) \sup _{0<j<k} M_{h+p(j+1), k-j}(\Psi, \omega \times \Lambda)
$$

where the constant $B$ does not depend on $\varphi$ in $\mathcal{E}(\omega \times \Lambda)$ and $P$ but only on $h, k, p, \alpha_{k}$, $\omega, \omega^{\prime}$.

The proof of Lemma 2 is an easy adaptation of the proof of Schwartz [21, p. 186-188]: there are only minor changes of notations due to the infinite dimensional case.

We recall now that $\rho$ is a nonzero holomorphic function on $\Omega$ which is a connected $M$-open subset of the b.v.s. $E$.

Let $\rho \mathcal{E}(\Omega)=\{\rho \varphi\}_{\varphi \in \mathcal{E}(\Omega)}$. Since $\rho$ is nonzero on any $M$-open subset of $\Omega, \rho \varphi=0$ in $\mathcal{E}(\Omega)$ implies $\varphi=0$ hence there exists the injective map:

$$
\begin{aligned}
& \mathcal{E}(\Omega) \quad \supset \quad \rho \mathcal{E}(\Omega) \quad \rightarrow \quad \mathcal{E}(\Omega) \\
& \rho \varphi \quad \varphi
\end{aligned}
$$

Let us equip $\rho \mathcal{E}(\Omega)$ with the topology induced by that of $\mathcal{E}(\Omega)$.

LEMMA 3. The above map is continuous.

Proof. Let $\mathcal{V}$ be a 0 -neighborhood in $\mathcal{E}(\Omega)$ that we may assume of the form

$$
V(K, L, \varepsilon, n)=\left\{\varphi \in \mathcal{E}(\Omega) \text { such that } \sup _{\substack{x \in K \\ x_{i} \in L \\ 0<i<n}}\left|\varphi^{(i)}(x) x_{1} \ldots x_{i}\right|<\varepsilon\right\}
$$

where $K$ is a strictly compact subset of $E$ contained in $\Omega$, where $L$ denotes a convex balanced strictly compact subset of $E$ and where $\varepsilon>0$ and $n \in \mathbf{N}$.

Let $K^{\prime}$ be a convex balanced strictly compact subset of $E$ such that $K$ is compact in the Banach space $E_{K^{\prime}}$ and that $K$ and $L$ are contained in $K^{\prime}$ (but $K^{\prime}$ is not necessarily contained in $\Omega$ ).

Let $x_{0}$ be an element of $K$ such that $\rho\left(x_{0}\right)=0$. In order to be in the notations of Lemma 1 let us assume (by translation) that $x_{0}=0$. Then if $g=\rho / E_{K^{\prime}} \cap \Omega$ Lemma 1 gives: $E_{K^{\prime}}=F \oplus \mathrm{Ce}$ and $g=P . J$ in a 0 -neighborhood $\Omega^{\prime}$ of $E_{K^{\prime}}$ and we have all the properties on $P$ and $J$ listed in Lemma 1 .

Let $\varphi$ be in $\mathcal{E}(\Omega)$ and let $\varepsilon_{0}>0$ such that $\varepsilon_{0} K^{\prime} \subset \Omega^{\prime}$. Let $\Lambda$ be an open 0 -neighborhood of $F$ ( $F$ is equipped with the topology induced by $E_{K^{\prime}}$ ) and $\omega$ an open 0-neighborhood of $\mathrm{C} e$ such that $\omega \times \Lambda \subset \varepsilon_{0} K^{\prime}$. Let us apply Lemma 2 to 
$P \cdot(J \varphi) / \omega \times \Lambda$ and $(J \varphi) / \omega \times \Lambda(J \varphi$ is here the function denoted by $\varphi$ in Lemma 2). Hence Lemma 2 gives us a local (in $E_{K^{\prime}}$ ) majorization of $J \varphi$ by means of a majorization of $P J \varphi$. Writing now explicitly the point $x_{0}$ for the convenience of the end of the proof we obtain: for every $n^{\prime \prime} \in \mathbf{N}$ and $\varepsilon^{\prime \prime}>0$ there exist $n^{\prime} \in \mathbf{N}$ and $\varepsilon^{\prime}>0$ such that

$$
\rho \varphi \in V\left(x_{0}+\varepsilon_{0} K^{\prime}, K^{\prime}, \varepsilon^{\prime}, n^{\prime}\right)
$$

implies

$$
\sup _{\substack{0<i<n^{\prime \prime} \\ x \in \omega^{\prime} \times \Lambda \\ y \in K^{\prime}}}\left|(J \varphi)^{(i)}\left(x_{0}+x\right) \cdot y\right| \leqslant \varepsilon^{\prime \prime} .
$$

((I) implies that

$$
\sup _{\substack{0<i<n^{\prime} \\ y \in K^{\prime} \\ x \in \omega \times \Lambda}}\left|\left[(P j \varphi)^{(i)}\left(x_{0}+x\right)\right] y\right| \leqslant \varepsilon^{\prime}
$$

which by Lemma 2 implies (II).) But $J$ is invertible on $x_{0}+\varepsilon_{0} K^{\prime}$ with $\varepsilon_{0}>0$ small enough; hence for every $n \in \mathbf{N}$ and $\varepsilon>0$ there exist $n^{\prime} \in \mathbf{N}$ and $\varepsilon^{\prime}>0$ such that $\rho \varphi \in V\left(x_{0}+\varepsilon_{0} K^{\prime}, K^{\prime}, \varepsilon^{\prime}, n^{\prime}\right)$ implies

$$
\varphi \in V\left(x_{0}+\mu K^{\prime}, K^{\prime}, \varepsilon, n\right)
$$

for some $\mu>0$ small enough. ((II) implies

which implies (III).)

$$
\sup _{\substack{0<i<n \\ x \in \mu K^{\prime} \\ y \in K^{\prime}}}\left|\varphi^{(i)}\left(x_{0}+x\right) \cdot y\right| \leqslant \varepsilon
$$

For an element $x_{0}$ of $K$ such that $\rho\left(x_{0}\right) \neq 0$ the above implication is trivially valid. Since $K$ is compact in $E_{K^{\prime}}$ the classical compacity technique implies Lemma 3.

Proof of TheOREM 1. It suffices to define a continuous linear form $l$ on $\rho \cdot \mathcal{E}(\Omega)$ by the formula $l(\rho \varphi)=T(\varphi)$. From Lemma 3 and the Hahn-Banach theorem there exists a continuous extension $S$ of $l$ to $\mathcal{E}(\Omega)$. Hence $S$ is in $\mathcal{E}^{\prime}(\Omega)$ and $\rho S=T$.

REMARK. An immediate consequence of Lemma 3 is that in the above conditions the ideal of multiples of $\rho$ is closed in $\mathcal{E}(\Omega)$.

4. Existence of solutions for the convolution equations in $\operatorname{Exp}\left(E^{\times}\right)$. Let $E$ be a complex $t$-separated Schwartz b.v.s. such that there exists a basis $\left(c_{i}\right)_{i \in I}$ of convex balanced bounded sets such that each $E c_{i}$ is a Banach space with the approximation property (this last property is well known to be true in any nuclear b.v.s. where the normed spaces $E c_{i}$ may be chosen separable Hilbert spaces from Pietsch [19, p. 65]: any nuclear map between Banach spaces may be factorized through a separable Hilbert space).

REMARK. Under these assumptions the Fourier-Borel transform

$$
\begin{array}{rl}
\mathcal{H}_{S}^{\prime}(E) \rightarrow \mathcal{F}_{\mathcal{H}}^{\prime}(E) & \subset \mathcal{H}\left(E^{\times}\right) \\
l & \mathscr{F} l: \varphi \rightarrow l\left(e^{\varphi}\right), \quad \varphi \in E^{\times},
\end{array}
$$

is injective. 
To prove this it suffices to show that the vector span of the set $\left|e^{\varphi}\right|_{\varphi \in E^{\times}}$is dense in $\mathcal{H}_{S}(E)$; it is proved in Colombeau, Meise and Perrot [7] that the finite type polynomials are dense in $\mathcal{H}_{S}(E)$; an easy proof (Boland [4, p. 51]) shows that each finite type polynomial is approximated by a combination of these exponentials.

Now $\mathcal{F}_{\mathcal{H}}(E)$ is described exactly as in Remark 3 of the introduction when $E$ is a nuclear b.v.s. (see Colombeau and Perrot [8]). We denote it by $\operatorname{Exp}\left(E^{\times}\right)$. If $E$ is a quasi-complete dual nuclear l.c.s. its von Neumann bornology makes it a nuclear b.v.s. It is proved in [8], [9] that $E^{\prime}$ is dense in $E^{\times}$; hence the two spaces $\operatorname{Exp}\left(E^{\prime}\right)$ and $\operatorname{Exp}\left(E^{\times}\right)$are exactly identical.

Now let us recall that a convolution operator on $\mathscr{F}_{\mathcal{F}}^{\prime}(\Omega)$ is by definition a linear continuous map from $\mathscr{F} \mathcal{H}_{S}^{\prime}(\Omega)$ into itself which commutes with the translations $\tau_{\alpha}$ for every $\alpha$ in $E^{\times}$.

As a corollary of Theorem 1 we recover the existence Theorem of [9] with a completely different proof.

THEOREM 2. Let E be a complex $t$-separated Schwartz b.v.s. such that there exists a basis $\left(c_{i}\right)_{i \in I}$ of convex balanced bounded sets such that each $E c_{i}$ is a Banach space with approximation property (in particular $E$ may be a nuclear b.v.s. and in this last case $\left.\mathscr{F}_{\mathcal{H}}^{\prime}(E)=\operatorname{Exp} E^{\times}\right)$. Let $\Omega \subset E$ be a convex balanced open set.

Then every nonzero convolution operator on $\mathscr{F}_{\mathcal{F}}^{\prime}(\Omega)$ is surjective.

Proof. It is known (Colombeau and Perrot [9, Lemma 2]) that any such convolution operator $\theta$ may be written $\theta=T *$ for some $T \in\left(\mathscr{F} \mathcal{H}_{S}^{\prime}(\Omega)\right)^{\prime}$. For a given $g$ in $\mathscr{F} \mathcal{H}_{S}^{\prime}(\Omega)$ let us then consider the equation $T * f=g$ which is equivalent to $\mathscr{F}^{-1}(T * f)=\mathscr{F}^{-1}(g)$ if $\mathscr{F}^{-1}$ is the inverse of $\mathscr{F}$. An easy verification shows that this equation becomes ${ }^{t} \mathscr{F}(T) \mathscr{F}^{-1}(f)=\mathscr{F}^{-1}(g)$ where ${ }^{t} \mathscr{F}$ is the transpose of $\mathscr{F}$ and where ${ }^{t} \mathscr{F}(T)$ is in $\mathcal{H}_{S}(\Omega)=\mathcal{H}_{S}^{\prime \prime}(\Omega)$ because $\mathcal{H}_{S}(\Omega)$ is Schwartz complete, hence semireflexive; see Colombeau, Meise and Perrot [7]. ${ }^{t} \mathcal{F}(T)$ is called the characteristic function of the operator $\mathcal{O} \mathscr{F}^{-1}(g)$ is in $\mathcal{H}_{S}^{\prime}(\Omega)$; hence (HahnBanach) may be continued as an element $h$ in $\mathcal{E}^{\prime}(\Omega)$. From Theorem 1 there exists a distribution $l$ in $\mathcal{E}^{\prime}(\Omega)$ such that ${ }^{t} \mathcal{F}(T) \cdot l=h$. The restriction of $l$ to $\mathcal{H}_{S}(\Omega)$ is in $\mathcal{H}_{S}^{\prime}(\Omega)$ and the restriction of $h$ to $\mathcal{H}_{S}(\Omega)$ is $\mathcal{F}^{-1}(g)$; hence the equation is solved.

5. On the solutions of the homogeneous equation $\theta f=0$. Let $E$ be a $t$-separated nuclear b.v.s. and let $\theta$ be a convolution operator on $\operatorname{Exp}\left(E^{\times}\right)$. From [9] $\theta=T$ * for some $T$ in $\left(\mathcal{F}_{\mathcal{F}} \mathcal{H}_{S}^{\prime}(E)\right)^{\prime} . \rho={ }^{t} \mathscr{F}(T) \in \mathcal{H}_{S}(E)$ is called the characteristic function of $\theta$ and the equation $\theta f=0$ is equivalent to

$$
\rho \cdot \mathscr{F}^{-1} f=0
$$

$\left(f \in \mathscr{F}_{\mathcal{S}}^{\prime}(E)\right.$; hence $\left.\mathscr{F}^{-1} f \in \mathcal{H}_{S}^{\prime}(E)\right)$.

TheOREM 3. Let E be a complex $t$-separated nuclear b.v.s. Let $\mathcal{O}$ be a convolution operator on $\operatorname{Exp}\left(E^{\times}\right)$and let $\rho \in \mathcal{H}_{S}(E)$ be its characteristic function. Then any solution $f$ in $\mathcal{F}_{\mathcal{H}} \mathcal{H}_{S}^{\prime}(E)$ of the homogeneous equation $\theta f=0$ is the Fourier-Borel transform of an element $U$ of $\mathcal{E}^{\prime}(E)$ such that $\rho U=0$. 
Remark 1. When $E$ is a nuclear l.c.s. it admits a base of Hilbertian 0-neighborhoods. In this last case one has trivially that for every open set $\Omega \subset E$ and every $x \in \Omega$ there is a $\varphi \in \mathcal{E}(E)$ with $\varphi(x)=1$ and supp $\varphi \subset \Omega$. Hence we may define the concept of support of an element of $\delta^{\prime}(E)$ as done in Guelfand and Chilov [14, $\S 1.4$, pp. 5, 6]. Then $\rho U=0$ implies that the support of $U$ is contained in the closure in $E$ of the set $\rho^{-1}(0)$ of the zeroes of $\rho$, which is precisely equal to $\rho^{-1}(0)$ if $E$ is a DFN or an FN space. We do not enter into more details on the concept of support of an element of $\mathcal{E}^{\prime}(E)$.

REMARK 2. It is known that in the finite dimensional case, Theorem 3 obtained in Gay [13] is much deeper than the usual approximation theorem. Let us just give a very particular case which helps to understand it: if $E=\mathbf{C}$ and $\theta f=\Sigma_{n>0} a_{n} f^{(n)}$ where $\rho(z)=\sum_{n \geqslant 0} a_{n} z^{n}$ is an entire function on $\mathbf{C}, \rho$ is the characteristic function of $\theta$.

Let $\left\{z_{k}\right\}_{k \geqslant 1} \subset \mathbf{C}$ be the set of zeroes of $\rho$ and let $m_{k} \geqslant 1$ be the multiplicity order of $z_{k}$. Every solution $f$ of the homogeneous equation $\theta f=0$ has the form

$$
f(z)=\sum_{1<j<m} P_{j}(z) e^{\alpha_{k_{j}} z}
$$

where $P_{j}$ is a polynomial of degree $\leqslant m_{k}-1$ and where $n \in \mathbf{N}$.

Now let us prove Theorem 3. Before the proof we need the following lemma. (Let $\mathcal{E}_{(0,1)}(E)$ be the space of the $C^{\infty}$ forms of type $(0,1)$ on $E$ and let $\mathcal{E}_{(0,1) \text {, closed }}(E)$ be the subspace of the closed forms (see [10] for details).)

LEMMA 4. Let $E$ be a complex Schwartz b.v.s. and $\Omega$ a connected M-open subset of E. Let $T \in \mathcal{E}_{(0,1) \text {, closed }}^{\prime}(\Omega)$ and $\rho \in \mathcal{H}_{S}(\Omega)$ with $\rho \neq 0$. Then there exists a $S \in$ $\mathcal{E}_{(0,1), \text { closed }}^{\prime}(\Omega)$ with $\rho S=T$.

Proof. We first prove that the following map is continuous.

$$
\begin{gathered}
\mathcal{E}_{(0,1), \text { closed }}(\Omega) \supset \rho \cdot \mathcal{E}_{(0,1) \text {, closed }}(\Omega) \rightarrow \mathcal{E}_{(0,1) \text {, closed }}(\Omega) . \\
\rho \varphi \rightarrow \varphi
\end{gathered}
$$

A fundamental system of 0 -neighborhoods in $\mathcal{E}_{(0,1) \text {, closed }}(\Omega)$ is made of sets of the form

$$
\begin{aligned}
& W(K, L, \varepsilon, n) \\
& \quad=\left\{\varphi \in \mathcal{E}_{(0,1), \text { closed }}(\Omega) \text { such that } \sup _{\substack{0<i<n, x \in K \\
x_{i} \in L, y \in L}}\left|\varphi^{(i)}(x)\left(x_{1} \ldots x_{i}\right) \cdot y\right|<\varepsilon\right\}
\end{aligned}
$$

if $K$ is a strictly compact subset of $\Omega, L$ a bounded subset of $E, \varepsilon>0$ and $n \in \mathbf{N}$. As usual let

$$
V(K, L, \varepsilon, n)=\left\{h \in \mathcal{E}(\Omega) \text { such that } \sup _{\substack{0<i<n \\ x \in K, x_{i} \in L}}\left|h^{(i)}(x)\left(x_{1} \ldots x_{i}\right)\right| \leqslant \varepsilon\right\} .
$$

For $y \in E$ and $\varphi \in \mathcal{E}_{(0,1) \text {, closed }}(\Omega)$ let us consider the map:

$$
\begin{aligned}
& \varphi_{y}: \Omega \rightarrow \mathrm{C} \\
& x \rightarrow \varphi(x) \cdot y
\end{aligned}
$$


Then $\varphi_{y} \in \mathcal{E}(\Omega)$ and $\varphi^{(i)}(x)\left(x_{1} \ldots x_{i}\right) y=\varphi_{y}^{(i)}(x)\left(x_{1} \ldots x_{i}\right)$. Hence $\varphi \in$ $W(K, L, \varepsilon, n) \Leftrightarrow \varphi_{y} \in V(K, L, \varepsilon, n)$ for every $y \in L$. Let now $W(K, L, \varepsilon, n)$ be given. From Lemma 3 there is a $V\left(K, L^{\prime}, \varepsilon^{\prime}, n^{\prime}\right)$ such that if $h \in \mathcal{E}(\Omega) \rho h \in$ $V\left(K^{\prime}, L^{\prime}, \varepsilon^{\prime}, n^{\prime}\right) \Rightarrow h \in V(K, L, \varepsilon, n)$. Hence from the equivalence above $\rho \varphi \in$ $W\left(K^{\prime}, L^{\prime}, \varepsilon^{\prime}, n^{\prime}\right) \Rightarrow \varphi \in W(K, L, \varepsilon, n)$ if furthermore $L^{\prime} \supset L$ which we may assume. This proves the continuity of the above map $\rho \varphi \rightarrow \varphi$. Now Lemma 4 is proved similarly to Theorem 1.

Proof of Theorem 3. First step. Let us assume $E$ is a DFN space, $\mathcal{E}_{(0,1)}(E)$ is a Fréchet-Schwartz space (see Colombeau [5] or Colombeau and Meise [6]) and $\mathcal{E}_{(0,1) \text {, closed }}(E)$ is a closed subspace of it, hence a Fréchet-Schwartz space also. From [10] we have the exact sequence of Fréchet-Schwartz spaces:

$$
0 \rightarrow \mathcal{H}_{S}(E) \stackrel{i}{\rightarrow} \mathcal{E}(E) \stackrel{\bar{\partial}}{\rightarrow} \mathcal{E}_{(0,1), \text { closed }}(E) \stackrel{\bar{\partial}}{\rightarrow} 0
$$

hence by transposition we have still an exact sequence:

$$
0 \leftarrow \mathcal{H}_{S}^{\prime}(E) \stackrel{{ }^{i}}{\leftarrow} \mathcal{E}^{\prime}(E) \stackrel{t \bar{\partial}}{\leftarrow} \mathcal{E}_{(0,1), \text { closed }}^{\prime}(E) \stackrel{t \bar{\partial}}{\leftarrow} 0 .
$$

Let $\mathscr{T}=\mathscr{F}^{-1} f ; \mathcal{T}$ is in $\mathcal{H}_{S}^{\prime}(E)$; since ${ }^{t} i$ is surjective there exists an $S$ in $\mathcal{E}^{\prime}(E)$ such that ${ }^{t} i(S)=\mathcal{T}$ (i.e. $S(h)=\mathcal{T}(h)$ for every $h$ in $\mathcal{H}_{S}(E)$ ).

Let $\rho \in \mathcal{H}_{S}(E)$ be the characteristic function of the operator $\theta . \rho S \in \mathcal{E}^{\prime}(E)$ and furthermore $\rho S \in(\operatorname{Im} i)^{\perp}$ (i.e. $\rho S$ is null on $\operatorname{Im} i \subset \mathcal{E}(E)$ ) because if $h$ is in $\mathcal{H}_{S}(E)$ :

$$
\langle\rho S, i h\rangle=\langle S, \rho i h\rangle=\langle\mathcal{T}, \rho h\rangle=\langle\rho \mathcal{T}, h\rangle=0 \quad \text { because } \rho \mathcal{T}=0 .
$$

$(\operatorname{Im} i)^{\perp}=\operatorname{Ker}^{t} i=\operatorname{Im}{ }^{t} \bar{\partial}$; hence there exists an element $Y$ of $\mathcal{E}_{(0,1) \text {, closed }}^{\prime}(E)$ such that $\rho S={ }^{t} \bar{\partial}(Y)$. Since $\rho$ is in $\mathcal{H}_{S}(E)$ it follows from Lemma 4 proved above that there exists an element $X$ of $\mathcal{E}_{(0,1) \text {, closed }}^{\prime}(E)$ such that $\rho X=Y$.

Let $U=S-{ }^{t} \bar{\partial} X \in \mathcal{E}^{\prime}(E) .{ }^{t} i(U)={ }^{t} i(S)$ because ${ }^{t} i \circ{ }^{t} \bar{\partial}=0$ (from the exact sequence above); hence $U(h)=S(h)$ for each $h$ in $\mathcal{H}_{S}(E)$; hence $U=\mathcal{T}$ in $\mathcal{H}_{S}^{\prime}(E)$; hence $f=\mathscr{F} U$.

Let us prove that $\rho U=0$. If $h \in \mathcal{E}(E)$ we shall prove that $\langle\rho U, h\rangle=0$. For this $\langle\bar{\partial} y, h\rangle=\langle\rho S, h\rangle=\langle S, \rho h\rangle$ and furthermore

$$
\left\langle{ }^{t} \bar{\partial} Y, h\right\rangle=\langle Y, \bar{\partial} h\rangle=\langle\rho X, \bar{\partial} h\rangle=\langle X, \rho \bar{\partial} h\rangle=\langle X, \bar{\partial} \rho h\rangle
$$

(the last equality is due to the fact that $\bar{\partial} \rho=0$ ); hence $\left\langle{ }^{t} \bar{\partial} Y, h\right\rangle=\left\langle{ }^{t} \bar{\partial} X, \rho h\right\rangle$.

Since $\langle U, \rho h\rangle=\langle S, \rho h\rangle-\left\langle{ }^{t} \bar{\partial} X, \rho h\right\rangle$ it follows that $\langle U, \rho h\rangle=0$ which ends the first step of the proof.

Second step in the proof. The general case for $E$. $E$ is now a nuclear b.v.s. separated by its dual. Using the technique developed at the end of the paper of the existence theorem of [9] there exists a family $\left(\varepsilon_{i}\right)_{i \in I}$ of nuclear Silva spaces such that $E$ is the bornological inductive limit of the spaces $\mathcal{E}_{i}$, that $\mathscr{F} \mathcal{H}_{S}^{\prime}(E)$ is the bornological inductive limit of the spaces $\left(\mathscr{F} \mathcal{H}^{\prime}\left(\mathcal{E}_{i}\right)\right)_{i \in I}$ and that, for each index $i$, the restriction of $\mathcal{O}$ to $\mathcal{F} \mathcal{H}^{\prime}\left(\mathcal{E}_{i}\right)$ has range in $\mathscr{F} \mathcal{H}^{\prime}\left(\mathcal{E}_{i}\right)$ and is a convolution operator on the space $\mathscr{F} \mathcal{H}^{\prime}\left(\mathcal{E}_{i}\right)$.

For each $i \in I$ we denote by ( $i)$ the injective canonical map:

$$
\mathscr{F} \mathcal{H}^{\prime}\left(\mathcal{E}_{i}\right) \stackrel{(i)}{\rightarrow} \mathscr{F} \mathcal{H}_{S}^{\prime}(E)
$$


Let $f$ be an element of $\mathcal{F}_{\mathcal{H}}^{\prime}(E)$ such that $\theta f=0$. There exists an index $i_{0} \in I$ and an element $f_{0}$ of $\mathscr{F} \mathcal{H}^{\prime}\left(\mathcal{E}_{i_{0}}\right)$ such that $f=\left(i_{0}\right)\left(f_{0}\right)$.

Hence from the first step of the proof there exists an element $U_{0}$ of $\mathscr{E}^{\prime}\left(\mathcal{E}_{i_{0}}\right)$ such that $\rho_{i_{0}} U_{0}=0$ where $\rho_{i_{0}} \in \mathcal{H}\left(\mathcal{E}_{i_{0}}\right)$ is the characteristic function of the restriction of $\mathcal{O}$ to $\mathscr{F} \mathcal{H}^{\prime}\left(\mathcal{E}_{i_{0}}\right)$ and such that $f_{0}=\mathscr{F} U$ [where here $\mathscr{F}$ maps $\mathcal{H}^{\prime}\left(\mathcal{E}_{i_{0}}\right)$ onto $\left.\mathscr{F} \mathcal{H}^{\prime}\left(\mathcal{E}_{i_{0}}\right)\right]$.

Let $\rho$ denote the characteristic function of the convolution operator $\theta$ on $\mathscr{F} \mathcal{H}_{S}^{\prime}(E)$.

REMARK. $\rho_{i_{0}}$ is the restriction of $\rho$ to $\mathcal{E}_{i_{0}}$.

Proof. On $\mathcal{H}_{S}^{\prime}(E)$ the operator corresponding to $\theta$ is that of multiplication by $\rho$, which induces on $\mathcal{H}^{\prime}\left(\mathcal{E}_{i_{0}}\right)$ the multiplication by the restriction of $\rho$ to $\mathcal{E}_{i_{0}}$.

Now we are going to end the Proof of Theorem 3.

Let

$$
\begin{array}{ccc}
r: \mathcal{E}(E) & \rightarrow & \mathcal{E}\left(\mathcal{E}_{i_{0}}\right) \\
\varphi & \varphi / \mathcal{E}_{i_{0}}
\end{array}
$$

denote the restriction map and let ${ }^{t} r$ be the transposed map:

$$
\begin{array}{ccc}
\mathcal{E}^{\prime}\left(\mathcal{E}_{i_{0}}\right) & \stackrel{{ }^{r}}{\rightarrow} & \mathcal{E}^{\prime}(E) \\
U & & {\left[{ }^{t} r(U)\right](\varphi)=U\left[\varphi / \mathcal{E}_{i_{0}}\right]}
\end{array}
$$

We have the following commutative diagram (where the two arrows on the right-hand side are not explicitly denoted).

$$
\begin{aligned}
& \mathcal{H}\left(\mathcal{E}_{i_{0}}^{\times}\right) \supset \mathscr{F} \mathcal{H}^{\prime}\left(\mathcal{E}_{i_{0}}\right) \stackrel{\mathscr{F}}{\leftarrow} \mathcal{H}^{\prime}\left(\mathcal{E}_{i_{0}}\right) \leftarrow \mathcal{E}^{\prime}\left(\mathcal{E}_{i_{0}}\right) \\
& \downarrow\left(i_{0}\right) \quad \begin{array}{ccc}
U_{0} & U_{0} \\
\downarrow^{t} r & \downarrow^{t} r
\end{array} \\
& { }^{\prime} r\left(U_{0}\right) \quad \operatorname{tr}\left(U_{0}\right) \\
& \mathscr{H}\left(E^{\times}\right) \supset \mathcal{F}_{\mathcal{H}}^{\prime}(E) \stackrel{\mathscr{F}}{\leftarrow} \mathcal{H}_{S}^{\prime}(E) \leftarrow \mathcal{E}^{\prime}(E)
\end{aligned}
$$

Let $U={ }^{t} r\left(U_{0}\right)$. Since $f_{0}=\mathscr{F} U_{0}$ we have $f=\mathscr{F}\left[{ }^{t} r\left(U_{0}\right)\right]=\mathscr{F} U$. It remains now to prove that $\rho U=0$ : if $\varphi \cdot \in \mathcal{E}(\Omega)$

$$
\langle\rho U, \varphi\rangle=\left\langle{ }^{t} r\left(U_{0}\right), \rho \varphi\right\rangle=\left\langle U_{0}, r(\rho \varphi)\right\rangle=\left\langle U_{0}, \rho_{i_{0}} r(\varphi)\right\rangle=0
$$

because $r(\rho)=\rho_{i_{0}}$ by the previous remark.

ACKNOWLEDGEMENTS. One of the authors is particularly indebted to L. Nachbin for his encouragements and his kind hospitality in Rio de Janeiro.

\section{REFERENCES}

1. V. I. Averbuh and O. G. Smolyanov, The various definitions of the derivative in linear topological spaces, Russian Math. Surveys 23 (1968), no. 4, 67-113.

2. P. J. Boland, Malgrange theorem for entire functions on nuclear spaces, Proceedings on Infinite Dimensional Holomorphy, Lecture Notes in Math., vol. 364, Springer, Berlin and New York, 1974, pp. 135-144.

3. Holomorphic functions on nuclear spaces, Publicaciones del Departamento de Analisis Mat., Univ. de Santiago de Compostela, (B) no. 16 (1976).

4. J. F. Colombeau, $C^{\infty}$ mappings in infinitely many dimensions and applications (preprint).

5. Sur les applications différentiables et analytiques au sense de J. Sebastiaõ e Silva, Portugal. Math. 36 (1977), 103-118. 
6. J. F. Colombeau and R. Meise, $C^{\infty}$ functions on locally convex and on bornological vector spaces, Advances in Functional Analysis, Holomorphy and Approximation Theory, (Ed., S. Machado), Lecture Notes in Math., Springer, Berlin and New York (in press).

7. J. F. Colombeau, R. Meise and B. Perrot, $A$ density result in spaces of Silva holomorphic mappings, Pacific J. Math. 84 (1979), 35-42.

8. J. F. Colombeau and B. Perrot, The Fourier-Borel transform in infinitely many dimensions and applications, Advances in Functional Analysis, Holomorphy and Approximation Theory, (Ed., S. Machado), Lecture Notes in Math., Springer, Berlin and New York (in press).

9. Convolution equations in spaces of infinite dimensional entire functions of exponential type, Trans. Amer. Math. Soc. 258 (1980), 191-198.

10. _ The $\bar{\partial}$ equation in DFN spaces, J. Math. Anal. Appl. (in press).

11. Th. A. W. Dwyer III, Differential operators of infinite order in locally convex spaces. I, Rend. Mat. 10 (1978), 149-179.

12. __ Differential operators of infinite order in locally convex spaces. II, Rend. Mat. 10 (1978), 273-293.

13. R. Gay, Sur un problème de division des fonctionnelles analytiques, C. R. Acad. Sci. Paris Sér. A 283 (1976), 835-838.

14. I. M. Guelfand and G. E. Chilov, Les distributions, Tome 1, Dunod, Paris, 1962.

15. H. Hogbe-Nlend, Bornologies and functional analysis, North-Holland Math. Studies, vol. 26 North-Holland, Amsterdam, 1978.

16. L. Hörmander, On the division of distributions by polynomials, Ark. Mat. 3 (1958), 555-568.

17. S. Lojasiewicz, Sur le probleme de la division, Studia Math. 18 (1959), 87-136; Rozprawy Matematyczne 22 (1961).

18. M. Z. Nashed, Differentiability and related properties, Nonlinear Functional Analysis and Applications, Academic Press, New York and London, 1971.

19. A. Pietsch, Nuclear locally convex spaces, Ergebnisse der Mathematik und ihrer Grenzgebiete, Bd. 66, Springer, Berlin and New York, 1972.

20. J. P. Ramis, Sous ensembles analytiques d'une variété Banachique complexe, Ergebnisse der Mathematik und ihrer Grenzgebiete, Bd. 53, Springer, Berlin and New York, 1970, pp. 103-309.

21. L. Schwartz, Division par une fonction holomorphe sur une variété analytique complexe, Summa Brasil. Math. 3 (1955), 181-209.

22. L. Waelbroeck, Some theorems about bounded structures, J. Funct. Anal. 1 (1967), 392-408.

23. __ Topological vector spaces and algebras, Lecture Notes in Math., vol. 230, Springer, Berlin and New York, 1971.

U.E.R. De Mathématiques et D'Informatique, Université de Bordeaux I, 33405 Talence, France 